\title{
Le bassin sidérurgique de Longwy : nouvelle reconversion, nouveaux acteurs
}

The steel basin of Longwy: new reconversion, new players

Das Stahlindustrierevier Longwy : neue Umgestaltung, neue Akteure

\section{Colette Renard-Grandmontagne}

\section{(2) OpenEdition}

\section{Journals}

Édition électronique

URL : http://journals.openedition.org/rge/1385

ISSN : 2108-6478

Éditeur

Association des géographes de l'Est

Édition imprimée

Date de publication : 1 juin 2006

ISSN : 0035-3213

Référence électronique

Colette Renard-Grandmontagne, «Le bassin sidérurgique de Longwy : nouvelle reconversion, nouveaux acteurs », Revue Géographique de l'Est [En ligne], vol. 46 / 3-4 | 2006, mis en ligne le 18 décembre 2009, consulté le 07 septembre 2020. URL : http://journals.openedition.org/rge/1385

Ce document a été généré automatiquement le 7 septembre 2020.

Tous droits réservés 


\section{Le bassin sidérurgique de Longwy : nouvelle reconversion, nouveaux acteurs}

The steel basin of Longwy : new reconversion, new players

Das Stahlindustrierevier Longwy : neue Umgestaltung, neue Akteure

Colette Renard-Grandmontagne

\section{Introduction}

1 Dans son édition longovicienne, Le Républicain Lorrain titrait récemment un article : « Il y a 25 ans débutaient les événements de Longwy » et invitait la population locale à un effort de mémoire, alors qu'une partie de celle-ci estime que « tout cela est de l'histoire ancienne et qu'il y a lieu de tourner la page... »'. Face à l'effondrement économique et humain que rappelle le journaliste avait été créé, le 19 juillet 1985, le PED (Pôle Européen de Développement) lors de la signature d'une Déclaration Commune Intergouvernementale par les trois États concernés (Belgique, France et Luxembourg). Pour symboliser le caractère innovant de ce projet transfrontalier et donner une impulsion forte à ce territoire traumatisé et anémié était ouverte la maison du PED, sur le Parc International d'Activités des Trois Frontières. Aujourd'hui, vingt ans plus tard, cette maison est fermée et Énergies, le journal de la communauté de communesde l'agglomération de Longwy fait de "l'ancien PED $»^{2}$, un outil temporaire de redynamisation de ce bassin sidérurgique. Une nouvelle étape semble donc franchie. Faut-il, d'ailleurs, pour souligner dès maintenant cette perception d'un changement, rappeler que le solde migratoire de la zone d'emploi de Longwy est redevenu positif au cours de la période 1999-2005 après avoir enregistré un déficit durant 40 ans (tableau 1)? 
Tableau 1 : Évolution de la population de la Zone d'Emploi de Longwy et de l'agglomération de Longwy

\begin{tabular}{|l|c|c|c|c|}
\hline & \multicolumn{2}{|c|}{ Zone d'Emploi de Longwy } & \multicolumn{2}{c|}{ Agglomération de Longwy* } \\
\hline & Nombre d'habitants & $\begin{array}{c}\text { Solde migratoire } \\
\text { durant chaque période } \\
\text { intercensitaire }\end{array}$ & Nombre d'habitants & $\begin{array}{c}\text { Solde migratoire } \\
\text { durant chaque période } \\
\text { intercensitaire }\end{array}$ \\
\hline 1962 & & & 53049 & n.d \\
1968 & 105154 & n.d & 54146 & -3606 \\
1975 & 94447 & n.d & 53404 & -4833 \\
1982 & 83881 & -12954 & 47069 & -9345 \\
1990 & 82700 & -2271 & 41300 & -7247 \\
1999 & $85000^{\text {*** }}$ & $+1176^{* *}$ & 40202 & -1884 \\
$2005^{* *}$ & & & n.d & n.d \\
\hline
\end{tabular}

* L'agglomération de Longwy est celle définie pour le recensement de 1982 qui a, aux 7 communes de la première Unité Urbaine (Longwy, Mont-Saint-Martin, Herserange, Lexy, Longlaville, Rehon et Saulnes) intégré la commune de Cosnes-et-Romain. Afın de permettre un suivi réel du solde migratoire au long de toute la période, la population de cette dernière commune a été ajoutée au nombre d'habitants de l'agglomération longovicienne pour les trois recensements antérieurs.

** Donnée parues dans AGAPE, Arrondissement de Briey - Les premiers résultats des recensements 2004 et 2005 de l'INSEE, Infobservatoire, n²0, mars 2006

Source: Tableau réalisé d'après les RGP de l'INSEE.

2 L'évocation rapide de ces quelques jalons chronologiques qui semblent se télescoperet qui pourtant s'inscrivent dans un quart de siècle - laisse entrevoir de profonds changements, de réelles réorientations. Ces dernières, pour la plupart récentes, sont au cœur de ce nouveau regard sur le territoire de Longwy qui a déjà suscité l'intérêt de nombreux géographes ${ }^{3}$. Ceux-ci ont surtout analysé le projet et les réalisations du PED ainsi que l'aide européenne à la reconversion. Cet article n'est en aucun cas une rétrospective, ni un bilan d'un quart de siècle de reconversion industrielle. Il cherche à éclairer la nature des réorientations les plus récentes, leur inscription dans les nouveaux paysages longoviciens et l'évolution du rôle des acteurs institutionnels et privés.

\section{De la reconversion par l'industrie à la diversification des activités : le rôle moteur du commerce}

Dans un espace dominé par les crassiers et organisé depuis des décennies autour des hauts fourneaux, des aciéries et des laminoirs qui occupaient les fonds des vallées de la Chiers et de la Moulaine et autour desquels étaient disposés les cités ouvrières et les bâtiments sociaux des usines, dans un territoire pétri de culture ouvrière et syndicale ne pouvaient succéder à la sidérurgie que des activités industrielles. Les "vrais emplois $»^{4}$ ne pouvaient être qu'industriels.

\section{A. Une difficile réindustrialisation}

Pendant plus de vingt ans, les efforts accomplis ont cherché à réindustrialiser le bassin par l'aménagement de nouvelles zones industrielles ou d'activités situées soit en terre vierge sur le plateau et desservies par l'axe rénové de la N52, soit sur une partie des anciens sites de production débarrassés de leurs friches industrielles, dépollués en surface et «renaturés » (photos 1 et 2). Le plus grand nombre des zones d'activités a pris place sur le plateau et ce dès 1969, avant même que fussent créées les zones dites 
"transitoires $»^{5}$ qui ont bénéficié durablement d'aides financières avantageuses par le biais de primes à l'installation à taux dérogatoire (34\% des investissements hors taxe).

Photo 1 : La friche de la Providence à Réhon en 1993

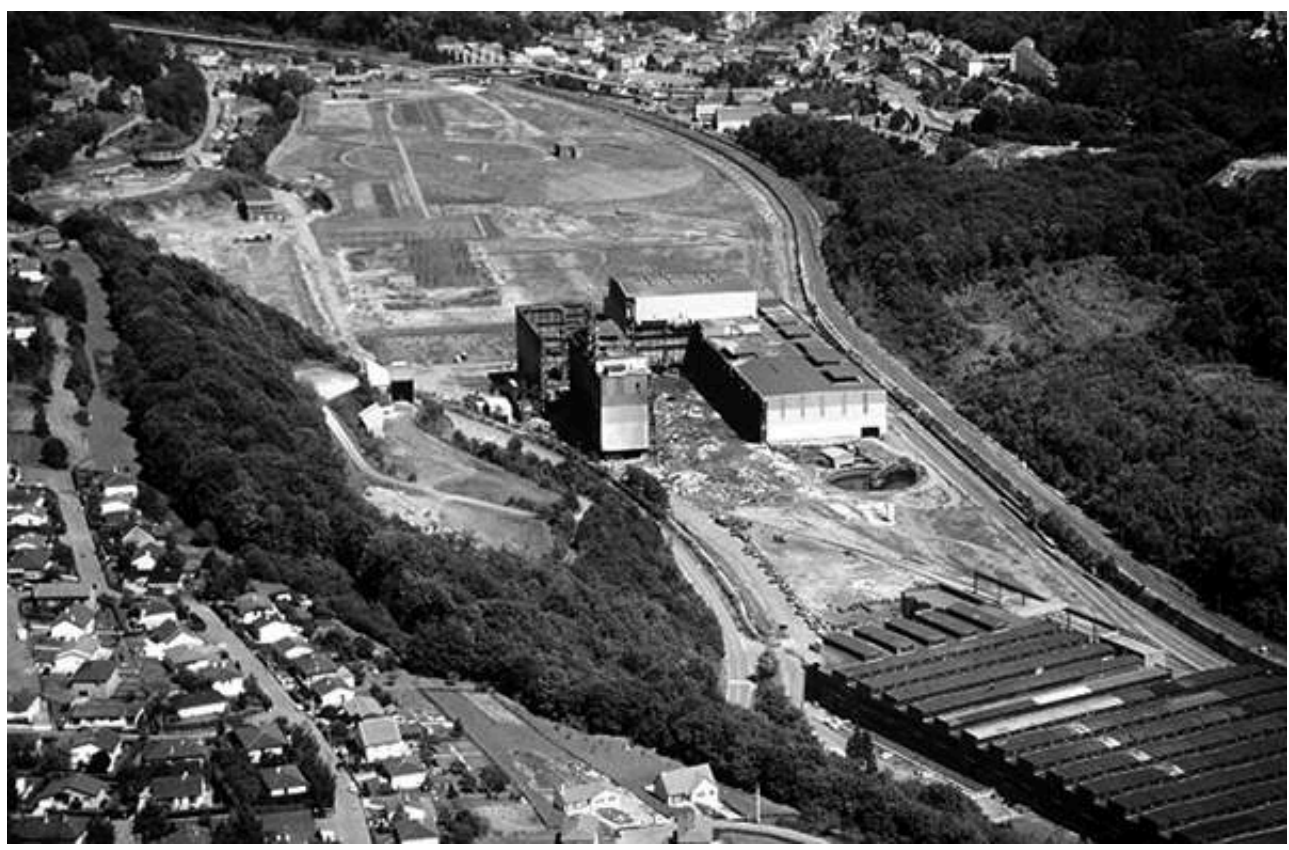

Le fond de la vallée de la Chiers, en aval de Longwy est, six années après l'arrêt des hauts fourneaux et de l'aciérie, en cours de requalification. La friche (70 ha) est en partie nettoyée. Subsiste, à gauche, au pied du crassier, le laboratoire aux façades noircies. Le premier plan est occupé par l'usine Lorraine Tubes de Lexy qui, en 2003, a été reprise par le sidérurgiste espagnol Condesa. Un second site de production est prévu à Lexy II. L'effectif du site resterait inchangé à environ 300 salariés.

Cl. A. Humbert 
Photo 2 : La friche « renaturée » de Réhon en juin 2006

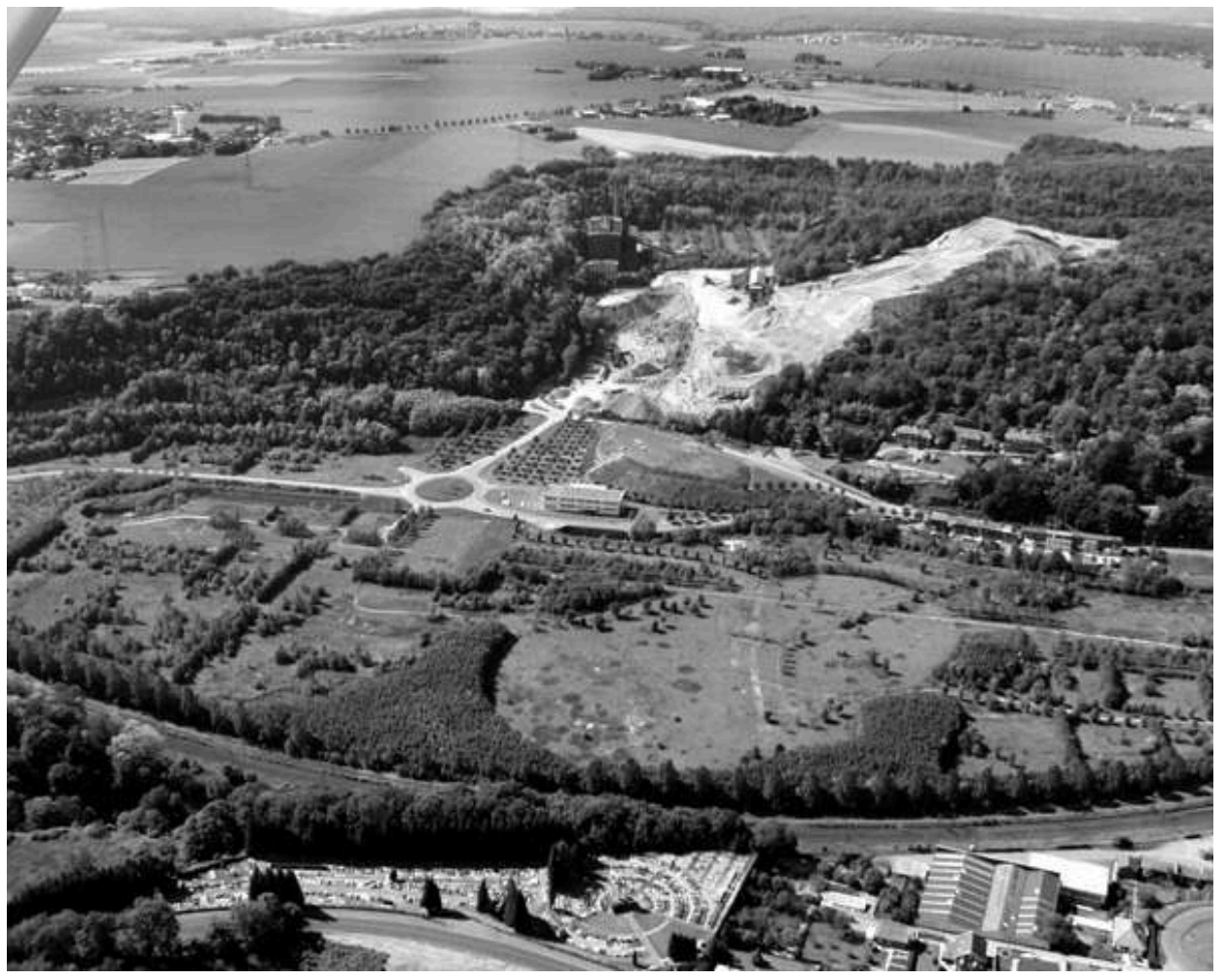

La friche de Réhon est maintenant photographiée perpendiculairement à l'axe de la photo 1, soit vers le nord-ouest, du cimetière de Réhon en direction du plateau couvert de céréales. Ne subsiste, essentiellement, qu'une partie du crassier déjà bien entamé par des prélèvements successifs. La partie inférieure a été terrassée et habillée pour servir de parking à l'hôtel intercommunal qui occupe I'ancien laboratoire entièrement rénové. En effet, les services de la CCAL (Communauté de Communes de l'Agglomération de Longwy) et les bureaux de l'AGAPE (Agence d'Urbanisme de l'Agglomération du Pôle Européen de Développement) s'y sont installés.

Cl. A. Humbert, C. Renard-Grandmontagne

5 La zone d'activités la plus vaste est celle du PIA (ParcInternational d'Activités) des Trois Frontières, créé en décembre 1988 et s'étendant sur 500 ha dont 280 ha pour la partie française qui correspond à l'emplacement des anciennes usines de la Chiers. L'aménagement de cette immense zone, une des plus vastes de Lorraine, constitua l'opération phare du PED. Celui-ci devait permettre, selon les objectifs fixés en 1985, l'accueil d'entreprises ou d'établissements devant créer 8000 emplois au total. Seules des entreprises extérieures à la région et/ou étrangères étaient susceptibles d'offrir ces emplois. Parmi les entreprises nationales, Peugeot s'est implanté à Villers-la-Montagne pour fabriquer des pièces de fonderie destinées à l'automobile. Parmi les entreprises étrangères, certaines se sont durablement installées telles que Performance Fibers ${ }^{6}$, d'autres n'ont été qu'éphémères; ce sont tout particulièrement les entreprises d'origine asiatique dont JVC localisé en 1988 à Villers-la-Montagne et qui, en octobre 1996, annonça la délocalisation de son site de production en Ecosse, Panasonic fermé au printemps $1998^{7}$ et plus récemment le coréen Daewoo dont les trois sites de production situés dans le nord de la Lorraine, ont été fermés en janvier $2003^{8}$. La fermeture de ces « usines-tournevis » a ravivé douloureusement les plaies encore mal cicatrisées des amputations précédentes et a généré de nouvelles friches. De ces bâtiments neufs qui ont brièvement porté l'espoir d'un renouveau industriel, seuls ceux de Panasonic et JVC sont actuellement réoccupés. Les deux bâtiments du bassin de 
Longwy ayant appartenu à Daewoo sont vides et celui de Mont-Saint-Martin, incendié, est même en cours de démolition. Cette seconde vague de fermetures est venue renforcer la difficulté pour la partie française d'atteindre l'objectif en terme d'emplois : 3 à 3500 ont été créés sur les 5500 prévus. Elle a contribué aussi à entretenir le vide persistant, certes verdoyant, des parcelles disponibles du PIA au sein de l'agglomération transfrontalière.

Ce cumul d'espérances déçues a très probablement participé à une prise de conscience progressive des difficultés qu'il y aurait à asseoir une reconversion par l'industrie dans un contexte national voire international de réduction des effectifs du secteur industriel, à capter et à retenir des projets industriels internationalement mobiles. Ce "tournant en marchant" a permis l'amorce d'une double réorientation pour le développement économique et la redynamisation de ce territoire. La plus spectaculaire est sans conteste l'ouverture du PIA à des activités autres qu'industrielles.

\section{B. L'ouverture du PIA au commerce}

7 Si la partie méridionale du PIA, la plus proche du cœur de Longwy-Bas a déjà accueilli des concessionnaires automobiles (Peugeot, Volkswagen, Opel, Fiat) pour faire de l'Espace Saintignon le pôle automobile local, c'est à Mont-Saint-Martin, à quelques encablures du «Point Triple», à la jonction des trois frontières, que l'opération commerciale la plus conséquente est amorcée. L'initiative émane d'un acteur économique privé issu de la grande distribution ; celui-ci a dû d'une part affronter les résistances locales et d'autre part lutter contre des concurrents voisins implantés en territoire belge et luxembourgeois dans un rayon de 10 à $15 \mathrm{~km}$.

En 1972 était ouvert dans cette localité, sur d'anciens terrains industriels, un hypermarché créé par les Coopérateurs de Lorraine. L'exploitation de cet hypermarché de $5255 \mathrm{~m}^{2}$ et de $1312 \mathrm{~m}^{2}$ de surface de vente dans la galerie marchande s'est faite successivement sous les enseignes suivantes :

1. de 1972 à 1986 : Rond Point,

2. de 1986 à 1992 : Euromarché,

3. de 1992 à 1998 : Mammouth,

4. depuis 1998 : Auchan.

Cependant, pour Mammouth d'abord, puis pour Auchan s'est imposée la nécessité de créer, sur une parcelle de 26 ha, un nouvel hypermarché beaucoup plus vaste ( $13000 \mathrm{~m} 2$ et $4700 \mathrm{~m} 2$ supplémentaires pour la galerie) par transfert du précédent devenu vétuste et obsolète. La réalisation de ce transfert fut longue ; en effet, envisagé en 1993, il n'a eu lieu effectivement qu'en septembre 2003 avec l'inauguration du centre commercial Pôle Europe, ceci en raison de la vigueur des résistances et de la difficulté à obtenir les autorisations réglementaires. La question écrite posée par Monsieur Ben Fayot (PSE) à la Commission Européenne, le 10 septembre 1998, est tout à fait révélatrice de l'attachement à une reconversion par l'industrie et de l'hostilité ouverte à tout projet autre qu'industriel. Nous reproduisons ici l'intégralité de la question écrite et la réponse donnée par madame Wulf-Mathieu au nom de la commission, le 28 octobre 1998. 
Document I : Question écrite E-2760/98 posée par M. Ben Fayot (PSE) à la commission (10 septembre 1998)

Objet : Implantation commerciale dans le pôle européen de développement (PED) L'implantation est envisagée d'une grande surface de plus de $18000 \mathrm{~m}^{2}$ (dont $13000 \mathrm{~m} 2$ pour un hypermarché et $5000 \mathrm{~m}^{2}$ pour une galerie commerciale) dans la partie française du pôle européen de développement. Ce centre important de distribution commerciale, qui vise la clientèle transfrontalière, exercera une pression énorme sur le commerce existant en France, en Belgique et au Luxembourg autour du PED.

La Commission peut-elle dire si le PED, créé sur des friches industrielles avec l'aide et l'apport financier de l'UE, peut servir de zone commerciale ou, si, selon la convention signée en 1995 par les partenaires, il ne devrait pas être plutôt destiné à créer de nouveaux emplois relevant de l'industrie ou du secteur des services industriels?

Ne faudrait-il pas pour le moins, dans un tel cas où la concurrence transfrontalière va jouer à plein, que les trois partenaires du PED (la France, la Belgique et le Luxembourg) s'entendent sur ce qui est acceptable pour chacun d'eux et ce qui ne l'est pas?

Enfin, la logique dans laquelle s'engage la partie française ne risque-t-elle pas à la fin du compte de détruire l'esprit de coopération européen qui était la base du PED?

La Commission entend-elle intervenir auprès de la France pour faire assurer le respect de l'objectif du pôle européen de développement et des finalités que l'UE a assignées à ce projet ?

Réponse donnée par Mme Wulf-Mathieu au nom de la Commission (28 octobre 1998) Le but du Pôle européen de développement (PED), tel qu'envisagé par les gouvernements français, belge et luxembourgeois dans la déclaration commune de 1985, était avant tout de reconvertir, par des actions communes, le bassin industriel transfrontalier de Longwy-Rodange-Athus, une zone sévèrement frappée par le déclin de l'industrie sidérurgique.

Les efforts auxquels s'est associée la Commission dès 1986, ont porté sur la reconstitution d'un tissu industriel diversifié, en mesure de remplacer l'industrie sidérurgique et d'assurer un nouveau développement économique du bassin.

Tout d'abord au travers des programmes nationaux d'intérêt communautaire, puis des programmes objectif II Lorraine et Luxembourg, la Communauté a cofinancé un régime d'aide aux investissements productifs, afin de stimuler les investissements des entreprises industrielles ou prestataires de services sur le site central du PED. L'implantation d'un grand centre de distribution n'entre pas dans le profil des investissements pouvant bénéficier d'un cofinancement communautaire. Toutefois, aucune disposition n'exclut l'implantation d'activités commerciales sur le Parc International d'Activités. La mise en œuvre du programme, et notamment le choix des projets, sont de la compétence des États membres.

Dans un esprit de coopération et afin de dégager un consensus, les trois États membres partenaires du PED ont entamé une concertation au sein de l'association transfrontalière de l'agglomération du pôle européen de développement. Cette Association, créée en 1996 et bénéficiant d'un cofinancement du Fonds Européen de Développement Régional dans le cadre du programme Interreg II, se veut en effet être le lieu de débat entre tous les acteurs publics ou privés concernés en vue de définir une politique commune d'aménagement du territoire des collectivités locales.

La Commission souhaite que l'esprit de coopération européen à la base du PED se perpétue, que le Parc international d'activités suive sa vocation initiale de zone d'accueil d'entreprises industrielles et que les trois partenaires aboutissent à une solution concertée.

Source : Journal officiel des Communautés européennes, 28 juin 1999, 1999/C 182/025. 

vocation initiale du PIA en tant que zone d'accueil d'entreprises industrielles, permet néanmoins une première ouverture vers un changement d'activités. Quant à l'autorisation d'exploitation, elle a requis plus de quatre années de procédures. Le 8 décembre 1999, la CDEC de Meurthe-et-Moselle ${ }^{9}$ a refusé cette autorisation au motif que le projet risquait de nuire au petit commerce; l'opposition n'émanait pas des membres du Pays Haut siégeant dans cette commission, mais était le résultat d'une hostilité de principe du représentant nancéien de l'UDAF (Union Départementale des Associations Familiales). Le rejet de ce projet, perçu comme un " diktat nancéien », une « injustice $~^{10}$, fut amèrement reçu par les Longoviciens et a probablement mobilisé davantage les élus et responsables locaux et cimenté les énergies pour soutenir le projet. Le 11 juillet 2000, Auchan a obtenu, en appel auprès de la $\mathrm{CNEC}^{11}$, une première autorisation pour créer cette surface de vente. CORA Belgique qui dispose d'un grand centre commercial de $27500 \mathrm{~m}^{2}$ a alors saisi le Conseil d'État d'un recours en annulation contre cette décision, en contestant notamment les composantes de la zone de chalandise présentées par Auchan. En effet, conformément à l'arrêt du 16 mars 1998 du Conseil d'État relatif aux Grands Magasins Ardennais, Auchan avait, dans son dossier de création par transfert, évalué d'une part l'importance quantitative de l'aire de chalandise nationale, c'est-à-dire française, et d'autre part pris en compte la clientèle belge et luxembourgeoise en l'assimilant à une clientèle touristique. En janvier 2003, malgré des conclusions favorables du Commissaire du gouvernement auprès du Conseil d'État, ledit Conseil a annulé la précédente autorisation considérant que, dans le nouveau contexte de constitution de la «zone euro", la zone de chalandise située à l'étranger devait être renseignée dans les mêmes conditions que celle du territoire français ${ }^{12}$. Tenant compte de ces prescriptions, Auchan a renouvelé sa demande d'autorisation auprès de la CNEC. Une autorisation définitive lui fut accordée le 9 septembre 2003, ce qui a permis l'ouverture du centre commercial au public le 20 septembre 2003.

11 La longue gestation de ce projet est à nouveau révélatrice des effets frontaliers contradictoires $^{13}$ dont bénéficie Longwy mais qui pénalisent aussi l'agglomération. Localisé à une des entrées de l'agglomération, mais aussi du territoire français, ce centre commercial baptisé «Pôle Europe » a été l'objet d'une recherche architecturale tant extérieure qu'intérieure conduite par Laurence Dutrieux (photos 3 et 4). 
Photo 3 : L'ouverture du PIA à la grande distribution (juin 2006)

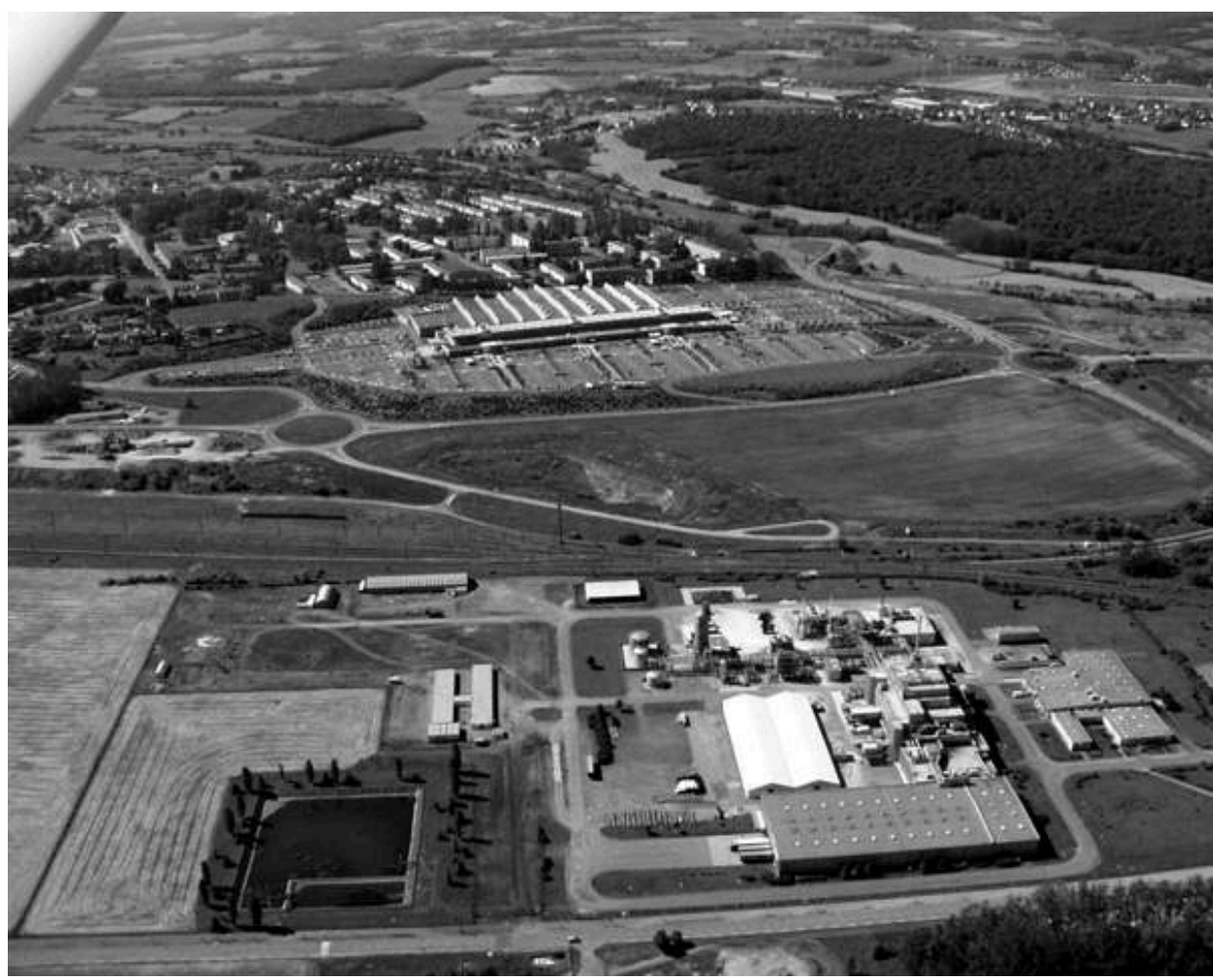

Cette photographie prise depuis Longlaville vers la ZUP de Mont-Saint-Martin, à quelques encablures du «Point Triple » rassemble deux symboles de la reconversion du bassin de Longwy. Au premier plan, l'usine américaine Performance Fibers représente la première phase caractérisée par l'implantation de grands établissements industriels extérieurs. Au second plan, le " paquebot » Auchan a fait l'objet d'une recherche architecturale soignée, comme en témoigne la photo 4.

Cl. A. Humbert, C. Renard-Grandmontagne 
Photo 4 : Façade de l'hypermarché Auchan (mars 2006)

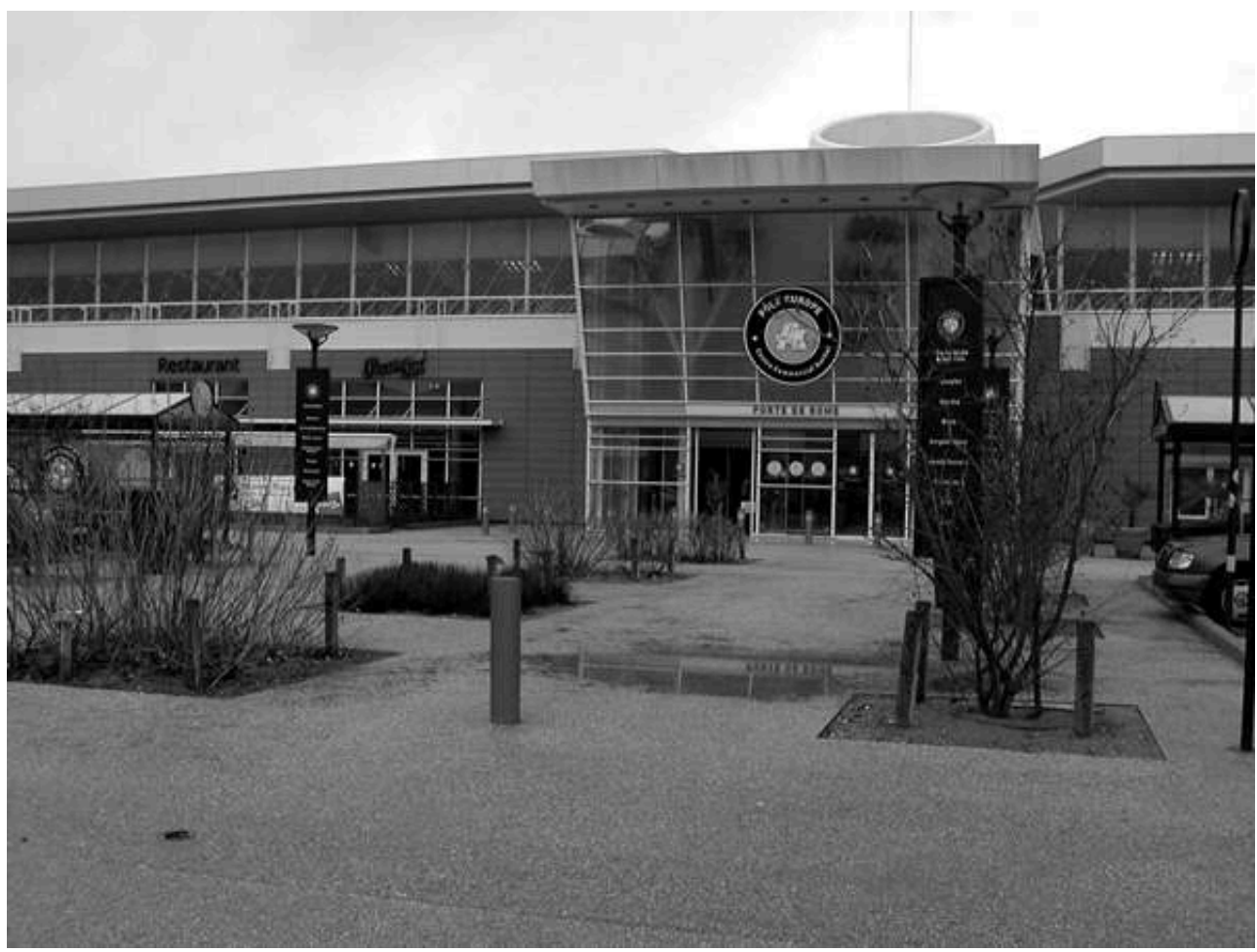

Cl. C. Renard-Grandmontagne

Le bâtiment - de forme parallélépipédique - a été intégré à la topographie locale en orientant sa plus longue façade parallèlement à l'axe de la vallée comme s'étire déjà Longlaville sur l'autre rive et en s'adaptant à la pente naturelle des Coteaux de MontSaint-Martin. Les aires de stationnement ont fait l'objet d'un traitement paysager sous la forme d'allées arborées et buissonnantes qui les cloisonnent. De plus, les alignements d'arbres évitent aux riverains la vue sur les parkings. Le choix des matériaux utilisés est réduit: terre cuite, verre et aluminium naturel. Ceux-ci fournissent l'essentiel des couleurs. Seule une laque verte a été appliquée sur les bardages. Esthétiquement, c'est une réussite qui peut contribuer à modifier l'image du Val Saint-Martin ${ }^{14}$, et à le réintégrer dans le tissu de la ville. Économiquement, le dossier déposé en CDEC, en 1999 et complété en 2003, affichait 307 Emplois à Temps Plein (ETP) sur l'ancien site et un effectif futur de 754 ETP (435 ETP pour l'hypermarché et 319 ETP pour la galerie marchande qui devait accueillir 47 commerces), soit l'embauche de 447 ETP dont 80 seraient réservés à des personnes en difficulté dans leur recherche d'emploi (public prioritaire pour l'ANPE) résidant dans le bassin de Longwy et plus particulièrement dans le quartier du Val. Comme la réglementation le prévoit, ce dossier devait aussi apprécier l'impact de la création sur l'emploi en calculant le solde des emplois. La différence entre le nombre des emplois prévus et celui des emplois susceptibles d'être supprimés s'élevait à 378 en 1999 et à 383 ETP en 2003. Selon les informations publiées dans le Guide 2006 de la distribution et celles recueillies oralement auprès des responsables d'Auchan et d'Immochan gestionnaire de la galerie marchande, les effectifs actuels sont d'environ 550 pour l'hypermarché et 278 ETP pour la galerie marchande, soit l'équivalent d'un grand établissement industriel. De plus, la diversité des cellules ouvertes dans le centre étoffe et renouvelle l'offre commerciale de 
l'agglomération de Longwy, dans son ensemble, en particulier dans le domaine de la restauration (8 restaurants).

Le «Pôle Europe » n'est peut-être que le premier élément, le noyau central d'un développement commercial encore plus ambitieux, voire plus audacieux dans cette partie du PIA ouverte sur les trois frontières. En effet, la société Immochan a acquis deux autres parcelles de $50373 \mathrm{~m}^{2}$ et $110678 \mathrm{~m}^{2}$ avec, respectivement, 4 et 8 ha utiles, situées de part et d'autre du centre commercial. La quatrième feuille de ce trèfle actuellement occupée par la friche Daewoo a été achetée, en 2004, par le groupe Antoine Frey, promoteur immobilier commercial mais aussi producteur de champagne ; il a obtenu, en mai 2005, l'autorisation d'ouverture de $18520 \mathrm{~m}^{2}$ de surfaces commerciales consacrées à l'équipement de la personne, dont une partie pour Decathlon.

Le projet d'évolution d'un centre commercial vers un "parc d'animations commerciales » tiré par une belle locomotive et situé à la porte d'entrée de la France est séduisant. C'est au total un projet de près de $45000 \mathrm{~m}^{2}$, de 450 emplois à temps plein nécessitant un investissement de 60 millions d'euros qui a été présenté aux élus locaux, conjointement par Immochan et le groupe Frey en novembre 2004 ${ }^{15}$. Sa réalisation peut néanmoins laisser dubitatif et autorise quelques interrogations relatives à l'équilibre des espaces marchands au sein de l'agglomération ${ }^{16}$, mais surtout à la rentabilité de ces éventuels nouveaux linéaires, tant l'équipement commercial de cet espace frontalier est devenu abondant.

La figure 1 rend compte de l'importance des surfaces commerciales actuelles et projetées dans le triangle Arlon-Longwy-Villerupt. Elle fait principalement ressortir la domination actuelle de la Belgique et la concurrence effrénée que se livrent les grandes enseignes de part et d'autre de la frontière franco-belge. Il semble bien que « la guerre des zones" ait commencé. De plus, des rumeurs alimentent cette impression de suréquipement commercial: celle d'un transfert-extension du petit hypermarché Carrefour d'Arlon à proximité du vaste Ikéa inauguré en 2005, et celle de l'ouverture d'un hypermarché Leclerc à Villerupt ; ceci dans une aire de chalandise transfrontalière évaluée entre 217000 et 260000 personnes, même si celles-ci ont un pouvoir d'achat un peu supérieur à la moyenne nationale. 
Figure 1 : Les pôles commerciaux de l'agglomération transfrontalière du Pôle Européen de Développement en 2006

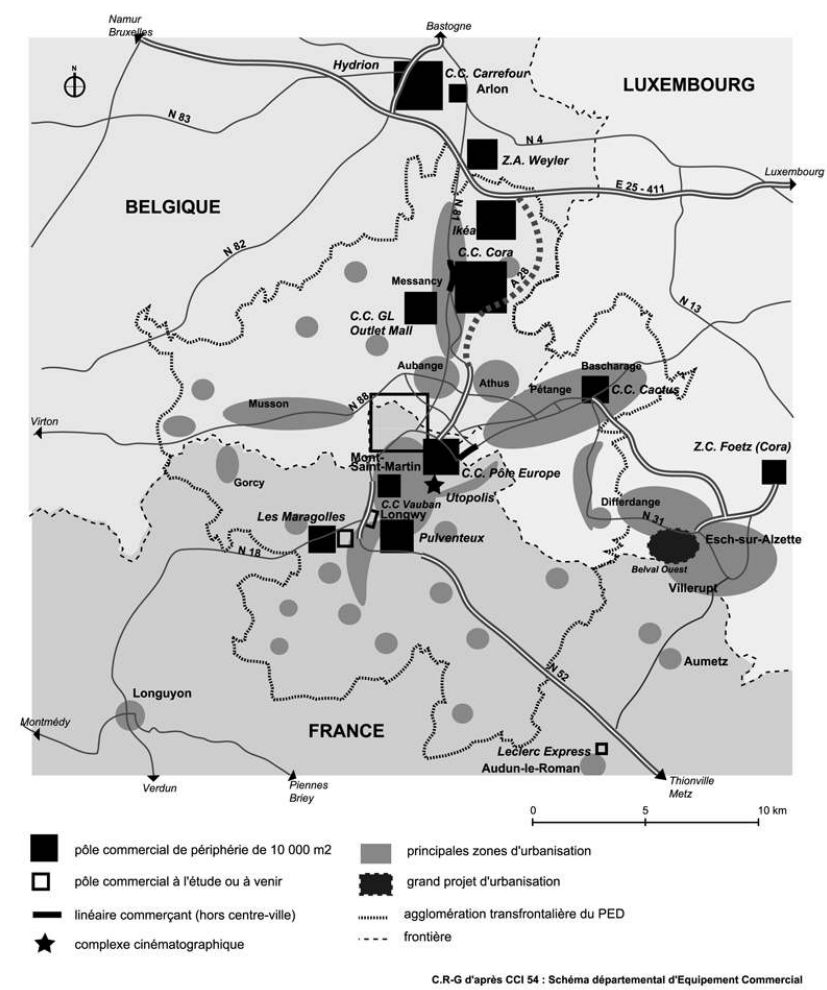

Si la frontière ne constitue plus un obstacle tarifaire, ni monétaire ${ }^{17}$, elle marque toujours cependant une limite entre différents dispositifs législatifs en matière d'autorisation d'ouverture de surfaces commerciales. Rappelons qu'en France la réglementation actuelle a été définie par deux lois successives : la loi Royer (1973) et la loi Raffarin (1996), cette dernière ayant rendu obligatoire la demande d'autorisation de création ou d'extension pour toute surface de vente supérieure à $300 \mathrm{~m}^{2}$ ainsi que l'indication de l'enseigne pour les superficies dépassant les $2000 \mathrm{~m}^{2}$. En Belgique, la réglementation est inspirée par celle en vigueur en France. La loi du 29 juin 1975, plus communément appelée « loi cadenas $»^{18}$, et ses textes d'application soumettaient à une autorisation préalable les projets d'implantations de commerce de détail selon des seuils supérieurs aux seuils réglementaires français. De plus, les procédures françaises sont considérées entre autres choses comme trop longues par les professionnels : « Une durée de deux à trois ans est fréquente, avec les recours. De tels délais sont incompatibles avec les réalités économiques ». Les procédures belges apparaissent plus brèves : de 5 à 6 mois dans les configurations les plus favorables, moins d'un an en cas de recours ${ }^{19}$. Comité socio-économique pour la distribution, commission provinciale et administration communale semblent donc plus réactifs que leurs homologues français. Néanmoins toutes les demandes n'étaient pas satisfaites comme en témoignent les résultats de l'année 1999. Au cours de cette année, 271 autorisations ont été délivrées portant sur une surface de vente de $322534 \mathrm{~m}^{2}$, alors que 127 demandes furent refusées, portant sur une surface de vente de $387177 \mathrm{~m}^{2}$. Quant au Grand Duché de Luxembourg, par la loi du 28 décembre 1988 modifiée par celle du 4 novembre 1997, tout projet de création, d'extension, de reprise, de transfert ou de changement d'activité d'un commerce d'une surface de vente supérieur à $400 \mathrm{~m}^{2}$ est soumis à une autorisation préalable du ministre des classes moyennes, après avis d'une 
commission d'équipement commercial. Mais la loi de 1997 a, de plus, suspendu pendant une durée de 5 ans, à compter de son entrée en vigueur, toutes les créations et extensions des centres commerciaux ou de magasins, spécialisés ou non, d'une surface de vente totale supérieure à $10000 \mathrm{~m}^{2}$. Ce moratoire est maintenant levé.

Ces différences réglementaires, certes mineures, mais néanmoins tangibles peuvent en partie expliquer l'avance prise par les pôles commerciaux belges avec lesquels devront compter les futures enseignes s'installant en France.

L'orientation commerciale acceptée par défaut dans un premier temps par les élus locaux est maintenant désirée car elle contribue à transformer l'image de l'agglomération dans laquelle le mot ouverture succède enfin à celui de fermeture. Elle participe sans aucun doute, aux côtés d'autres réalisations - telles que l'ouverture du cinéma Utopolis, de la médiathèque intercommunale au Pulventeux (Longwy) ou d'autres projets adoptés récemment comme celui d'un golf à l'emplacement des friches Senelle à Herserange - à développer l'attractivité du territoire dont les acteurs préfèrent désormais se rapprocher du Luxembourg plutôt que du sillon mosellan comme cela avait prévalu depuis le début de la crise.

19 La deuxième mutation récente très perceptible est celle de la prise en main du destin du territoire par les collectivités territoriales locales.

\section{De l'intervention forte de l'état a la prise en main du destin du bassin par les collectivités locales}

Le quart de siècle qui sert de cadre à cette étude peut, du point de vue des acteurs institutionnels c'est-à-dire des décideurs et des bailleurs de fonds, être subdivisée en deux phases principales. Pendant une longue période, les collectivités se sont peu impliquées dans les problèmes de reconversion et en ont été tenues à l'écart par les représentants de l'État. L'engagement des acteurs locaux est relativement récent. L'extrême fin du XXe siècle et le début des années 2000 constituent une période de transition.

\section{A. L'État français et l'Europe au chevet de l'ancien bassin sidérurgique}

Durant de nombreuses années, les acteurs locaux (élus, syndicalistes) ont analysé de façon très différente les causes du démantèlement de la sidérurgie sans parvenir véritablement à se projeter dans l'avenir avec une stratégie claire. Des oppositions politiques classiques entre élus de gauche et de droite au sein du conseil du district urbain, mais aussi des confrontations internes durables au sein de la gauche (PC-PS, CGT-CFDT) ont ralenti voire paralysé la conception d'une stratégie commune et l'élaboration d'un projet global. Rappelons, pour mémoire et de manière peut-être un peu trop simplifiée, que pour le Parti Communiste dominant, les principaux responsables de la « casse de la sidérurgie » étaient « l'Europe de Monsieur Davignon » et l'État français. De ce fait, l'État devait réparer mais les membres du PC étaient aussi hostiles au concept du PED qui ne constituait, de leur point de vue, que «le replâtrage d'une trahison $»^{20}$. Face à cette incapacité des élus à s'entendre sur un programme et face à l'effondrement financier des collectivités locales, c'est la stratégie de 
développement initiée, du côté français, par la Mission Interministérielle présidée le plus souvent par un représentant de l'État qui a prévalu, marginalisant les acteurs locaux. Durant 14 ans (1985-1999), l'État a été présent pour tenter de redynamiser l'exbassin sidérurgique. L'importance de l'action de l'EPFL (Établissement Public Foncier de Lorraine ${ }^{21}$ en est une des manifestations. Cet établissement public à caractère industriel et commercial a pour vocations la réalisation d'opérations foncières pour le compte de l'État et des collectivités locales et la prestation de services pour requalifier des espaces dégradés (anciens sites de production, quartiers urbains en déclin, cités ouvrières et, depuis le milieu des années 1990, sites militaires désaffectés). Mais il peut être aussi maître d'ouvrage, ce qui a été le cas pour le PIA des Trois Frontières et pour les sites reconvertis de Pompey-Frouard. Ces dernières opérations d'aménagement ont été entièrement financées par des subventions. Ainsi le financement total de l'aménagement de la ZAC du PIA (partie française, soit 280 ha) s'est élevé à 91 millions d'euros $^{22}$ (soit 600 millions de francs) qui provenaient pour :

1. $49 \%$ du FEDER,

$2.32 \%$ de l'État Français,

3. $10 \%$ des recettes internes de la ZAC,

$4.5 \%$ du Conseil Général de Meurthe-et-Moselle,

$5.4 \%$ de la Région Lorraine.

On remarquera l'absence totale de participation financière des collectivités territoriales locales au financement de la ZAC du PIA. Cependant, les acteurs locaux ont manifesté leur intérêt en participant à une « Association des Maires et Bourgmestres du PED» qui a créé une agglomération transfrontalière dotée depuis 1994 d'un Observatoire de l'Urbanisme. L'action d'aménagement menée par l'EPF Lorraine (à la fin de l'année 2005) peut se résumer sommairement comme suit :

- requalification du site de la partie française du PIA ;

- réalisation des infrastructures primaires et secondaires (VRD);

- réhabilitation de $13000 \mathrm{~m}^{2}$ d'anciennes halles industrielles ;

- traitement du lit et des rives de la Chiers avec en particulier sa mise à ciel ouvert au niveau du pôle des services ;

- constitution de 2302 ha environ de parcelles ou plates-formes viabilisées avec notamment :

- $\circ$ la plate-forme du Parc Central dont 35 ha acquis par la société Performance Fibers ;

- la plate-forme Belgique sud-est de 10,5 ha acquis par la société Daewoo-Orion ;

- les Hauts coteaux de Mont Saint Martin de 23,5 ha acquis par Auchan;

- les plates-formes du Petit Breuil (Photo 5) et du Prieuré pour une surface de 9 ha occupées par K. Kontainer (société islandaise de conteneurs à l'usage du fret aérien), le Centre Régional pour l'Innovation et le Transfert de Technologie (CRITT) et le pôle artisanal ;

- les plates-formes de Saintignon et de Gouraincourt de 10,5 ha où se sont implantés les concessionnaires automobiles déjà mentionnés ainsi qu'Utopolis ;

- les plates-formes Belgique nord-ouest (5 ha), des Bas Coteaux de Mont-Saint-Martin (8 ha), de l'Entre Deux Voies (20 ha) encore disponibles. 
Photo 5 : Plate-forme du Petit Breuil à Longwy en 2005

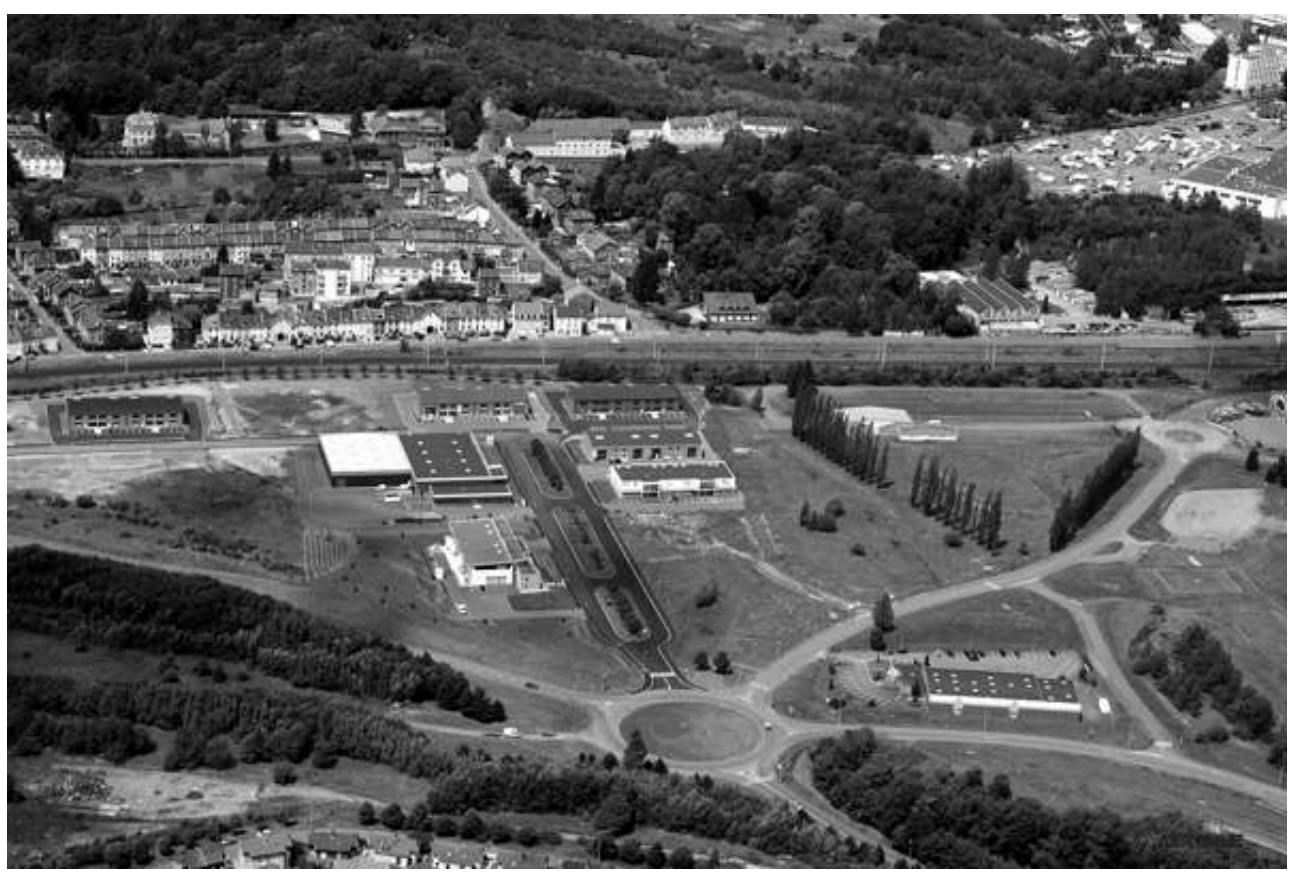

Sur cette plate-forme de 6 ha du PIA, en avant de la cité ouvrière de Gouraincourt, ont été construits plusieurs petits bâtiments hébergeant des artisans locaux ; chaque halle abrite quatre cellules d'une surface de $240 \mathrm{~m}^{2}$. Cette plate-forme symbolise l'attention portée récemment par la CCAL au développement endogène à partir de PME.

Cl. Aérovue/CCAL

Progressivement au cours de la deuxième moitié des années 1990, les élus locaux ont pris conscience de la valeur marchande des terrains maintenant viabilisés du PIA, notamment dans le cas de transactions destinées à des implantations commerciales ${ }^{23}$ et donc de la possibilité d'alimenter substantiellement les finances locales par des ressources éventuellement importantes.

\section{B. Désengagement de l'État, engagement des collectivités territoriales dans le cadre de l'intercommunalité}

Réagissant à la proposition d'acquisition d'une vaste parcelle par l'hypermarché de Mont-Saint-Martin présentée dans la première partie, les élus ont manifesté leur volonté de "récupérer leurs terrains ». L'État, par son préfet de Région, a saisi cette opportunité pour se désengager. La prise de distance de l'État s'est opérée dans la ZAC du PIA mais aussi dans l'ensemble du bassin de Longwy.

Suite aux décisions du CIADT (Comité Interministériel d'Aménagement et du Développement du territoire) du 18 mai 2000, des négociations furent engagées entre les partenaires concernés en vue d'une cession des terrains du PIA à la collectivité locale à la condition que soit réalisée une intercommunalité élargie. Le 21 décembre 2000 a été signé un protocole entre l'État, le District de l'Agglomération de Longwy constitué alors de 17 communes et l'EPF Lorraine. Il définit les modalités d'achèvement de la ZAC et le transfert de l'ensemble du patrimoine à titre gratuit audit district. Entre décembre 2000 et mars 2005 ont été signées, au fur et à mesure des demandes, plusieurs cessions anticipées de terrains. Le 31 décembre 2003 était fermée l'antenne de l'EPFL à 
Longwy et le 25 mars 2005, la signature de l'acte notarié scellait le transfert de l'intégralité du patrimoine du PIA au profit de la CCAL (Communauté de Communes de l'Agglomération de Longwy). La fin d'une ère !

À l'échelle du bassin de Longwy, celui-ci a obtenu dans le cadre du contrat de plan ÉtatRégion (2003-2007) un contrat territorial financé par l'État à hauteur de 91 millions d'euros ${ }^{24}$, soit l'équivalent du montant du financement de l'aménagement de la ZAC du PIA. L'effort durable réclamé par les socialistes locaux lors du paroxysme de la crise se poursuit effectivement, mais maintenant dans le cadre d'une intercommunalité en construction.

\section{La CCAL : une intercommunalité difficile à construire}

Succédant au premier district créé en 1960 (6 communes), élargi en 1999, la CCAL (18 communes, 55432 habitants) est née le $1^{\mathrm{er}}$ janvier 2002. C'est une construction à petits pas et encore incomplète. Alors que le nombre total d'habitants aurait pu autoriser une communauté d'agglomération instituant ipso facto une taxe professionnelle unique (TPU) sur l'ensemble du territoire intercommunal, la population de la ville-centre (14 521 habitants en 1999), très sensiblement en dessous du seuil réglementaire de 15000 habitants, ne l'a pas permis, semble-t-il. La loi Chevènement du 12 juillet 1999 sur l'intercommunalité offrait la possibilité pour les communautés de communes d'adopter une TPU. Cette option, fortement encouragée par l'État et fréquemment choisie par les communautés de communes les plus importantes, n'a pas été retenue par la CCAL. Seule une TPZ (taxe professionnelle de zone) de 7, 58 \% est appliquée aux entreprises implantées dans les zones pour lesquelles la CCAL est intervenue. Il est assez évident qu'une fois de plus, parmi d'autres obstacles, l'existence des zones d'activités constitue une entrave à la réalisation de l'intercommunalité. La zone d'activités la plus sensible est celle de Villers-la-Montagne déjà évoquée au début d'article. Cette zone rassemble environ 25 établissements, offre 2000 emplois et procure à la commune de Villers-la-Montagne plus d'un million d'euros, soit près de $80 \%$ de ses recettes fiscales. Son taux de taxe professionnelle est de 6,41 \% (2005), c'està-dire bien inférieur à ceux de Longwy ou de Mont-Saint-Martin. Comme d'autres communes françaises bien dotées en zones d'activités ${ }^{25}$, Villers-la-Montagne résiste à l'intercommunalité voulue par la ville-centre. Consciente de la difficulté de faire cavalier seul dans un territoire national où les structures intercommunales se sont considérablement développées depuis le début du XXI ${ }^{\mathrm{e}}$ siècle, Villers-la-Montagne cherche quelle stratégie développer pour échapper à ses voisins, lesquels, de plus, sont de couleur politique différente. Des mouvements réciproques de bonne volonté sont encore nécessaires pour rapprocher des communes qui, par ailleurs, appartiennent toutes à l'agglomération transfrontalière du PED.

Pour qui a survolé ou parcouru Longwy il y a plus de 25 ans et revient dans la " cité des émaux " aujourd'hui, le changement est considérable. Les paysages industriels sont métamorphosés : les couleurs dominantes ont été modifiées, la densité du bâti dans les fonds de vallées a cédé la place à une très grande aération. Fonctionnellement, cette forteresse industrielle et ouvrière de la Lorraine sidérurgique, ce "Texas français", cherche aujourd'hui à combler son déficit en équipements tertiaires et à développer des aménagements pour rendre attractif ce territoire davantage ouvert désormais sur les Trois Frontières, et plus particulièrement tourné vers le Luxembourg. Sa situation 
frontalière en fait un espace privilégié dans lequel les flux de travailleurs et de clients sont nourris, mais aussi un espace où les concurrences transfrontalières sont exacerbées. Le bassin de Longwy a été pénalisé en matière d'implantations industrielles au moment de la première phase de reconversion. Aura-t-il la possibilité de saisir la seconde chance que constituent les implantations commerciales?

Financièrement, les collectivités locales ont connu un état d'anémie extrême. Elles ont été placées sous perfusion grâce au versement de subventions essentiellement européennes et nationales. La manne européenne s'est tarie en 2001 avec la fin des engagements communautaires. D'autres perfusions ont pris le relais dans le cadre du contrat territorial et du contrat de site signé en septembre 2003 (17 millions d'euros). Mais ces crédits seront désormais gérés par les collectivités locales.

Le deuil de la sidérurgie a été long, très long. Il semble aujourd'hui terminé. Une nouvelle ère s'ouvre avec de nouvelles stratégies.

\section{BIBLIOGRAPHIE}

AïT OUMEZIANE A. (2000). - « Reconversion et construction d'un territoire transfrontalier : l'agglomération transfrontalière du pôle européen de développement des Trois Frontières (Belgique-France-Luxembourg) ", Annales de Géographie, n 611, p. 65-83.

HARDOUIN-LEMOINE M. (2001). - « Le bassin de Longwy : quelle reconversion grâce au programme européen Objectif 2 ? (1989-1996) », Annales de Géographie, n 619, p. 309-320.

NONN H., HÉRAUD J.-A. - dir. (1995). - Les économies industrielles en France de l'Est. Tissus et réseaux en évolution, Strasbourg, Presses Universitaires de Strasbourg, 305 p.

Panorama Trade Dimensions (2006). - Le guide 2006 de la Distribution, 1072 p.

Schulz ch. (1996). - « L'agglomération transfrontalière du pôle européen de développement (PED Longwy-Rodange-Athus) », Revue Géographique de l'Est, nº 2, p. 133-149.

SchULz ch. (1996). - Der Pôle Européen de Développement Longwy-Rodange-Athus (PED). Reaktivierung von Industriebrachen und wirtschaftliche Konversion, Université de Duisbourg.

Schulz ch. (1998). - «L'intercommunalité sans frontières ? État actuel et perspectives de la coopération transfrontalière des collectivités locales dans l'espace Saar-Lor-Lux ", Mosella, Tome XXIII, $\mathrm{n}^{\circ} 1-2$, p. 1-22.

Thull P. (2003). - Demain, la Lorraine, Éditions de l'Est, 222 p.

USVALD L. (1995). - Le pôle européen de développement de Longwy : un processus original de réindustrialisation?, Université de Nancy 2, Mémoire de maîtrise, 148 p. 


\section{NOTES}

1. Le Républicain Lorrain, édition de Longwy, 14 avril 2004. Les événements auxquels il est fait référence sont ceux de décembre 1978 déclenchés par l'annonce du nouveau plan social ramenant les effectifs dans la sidérurgie de 7700 à moins de 3000 personnes.

2. Énergies, journal de la CCAL (Communauté de Communes de l'Agglomération de Longwy), nº 08, mars 2006.

3. L'essentiel de ces contributions figure dans la bibliographie ci-dessous.

4. Expression employée par Jean-Paul Durieux, Président de la CCAL qui a été aussi maire de Longwy de 1989 à 2006 et député de la circonscription de 1993 à 2002.

5. Les zones dites «transitoires " sont des zones d'activités qui ont été aménagées avant même l'effondrement de la sidérurgie ou dans l'attente de la réhabilitation des friches industrielles de la vallée de la Chiers, afin d'offrir des terrains viabilisés à de nouveaux établissements susceptibles de participer à la reconversion. Il a été décidé que trois zones au plus, par pays, ne dépassant pas 50 ha chacune, pourraient bénéficier d'aides financières avantageuses. Les trois zones aménagées dans ce cadre, dans le bassin de Longwy, sont celles de Villers-la-Montagne, de Cosnes-et-Rosmain et de Gorcy.

6. L'actuel établissement Performance Fibers, d'origine américaine, s'est installé sur le PIA en 1991 sous le nom d'Allied Signal Industrial Fibers. Cette unité, en fonction depuis 1993, produit des fibres polyesther utilisées dans le renforcement des pneumatiques; elle emploie environ 300 salariés.

7. La Lorraine s'insurge contre les « chasseurs de primes », Le Monde, 22 octobre 1996 ; « Longwy revit les fermetures », Le Monde, 18 juillet 1998.

8. Le Coréen Daewoo s'est installé :

- en 1989, à Villers-la-Montagne pour produire des fours micro-ondes (300 salariés) ;

- en 1993, à Fameck, pour monter des téléviseurs (300 salariés)

- en 1995, à Mont-Saint-Martin, sur le PIA. Cette unité de production de tubes cathodiques pour téléviseurs employait 610 personnes.

Deux autres implantations avaient été annoncées : une usine de verre à Longwy et une unité de production de réfrigérateurs à Verdun.

9. CDEC : Commission Départementale d'Équipement Commercial présidée par le Préfet qui ne prend pas part au vote. Elle est constituée de 6 membres : 3 élus locaux (le maire de la commune d'implantation, un représentant de l'établissement public de coopération intercommunale, le maire représentant la commune la plus peuplée de l'arrondissement autre que la commune d'implantation), 2 représentants des activités commerciales et artisanales (le président de la CCI et le président de la Chambre des Métiers concernés par le projet) et un représentant des associations de consommateurs du département. La proposition de loi «Garantir l'équilibre des différentes formes de commerce ", présentée par le sénateur Alain Fouchéet adoptée par le Sénat le 16 juin 2005, propose de modifier, dans le Titre II, article 21, la composition de la commission en ajoutant le président du Conseil Général ou son représentant, en stipulant qu'ils ne doivent pas être élus de l'arrondissement d'implantation ni dans l'intercommunalité concernée.

10. Expressions respectivement de Frédéric Brigidi (maire communiste de Mont-Saint-Martin de 1977 à 2004, un des premiers soutiens à ce projet commercial) et d'Edouard Jacque (représentant du district de Longwy à la CDEC du 8 décembre 1999, maire de Cons-laGrandville, conseiller général du canton de Mont-Saint-Martin et député UMP de Longwy depuis 2002). On pourra lire l'expression de cette irritation dans deux articles du Républicain Lorrain : « Honteux, révoltant mais heureusement pas définitif », 12 décembre 1999 et « L'incompréhension fait place à une légitime indignation »,15 décembre 1999.

11. CNEC : Commission Nationale d'Équipement Commercial créée par la loi du 29 janvier 1993 était composée de 7 membres (3 hauts fonctionnaires, un magistrat de la Cour des Comptes et 3 
personnalités qualifiées). Depuis la loi Raffarin, les personnalités qualifiées sont au nombre de quatre. La proposition de loi citée en note 9 porte désormais sa composition à 9 membres nommés, pour une durée de 6 ans non renouvelables tout en renouvelant la commission par tiers tous les deux ans. La nouvelle composition proposée est la suivante :

- trois personnalités qualifiées, désignées par le président du Sénat, le président de l'Assemblée Nationale et le président du Conseil Économique et social,

- trois personnalités qualifiées, non-fonctionnaires, désignées par les trois ministres les plus concernés par l'équipement commercial : le ministre du commerce, celui de l'équipement et celui de l'aménagement du territoire,

- trois hauts fonctionnaires issus des corps d'inspection générale : l'inspection générale des finances, l'inspection générale de l'équipement et l'inspection générale du commerce et de l'industrie.

En moyenne, la CNEC accorde $5 \%$ des demandes d'ouverture.

12. Nouvel arrêt du Conseil d'Etat relatif à la délimitation de la zone de chalandise en région frontalière de janvier 2003: "La zone de chalandise décrite par le pétitionnaire (en l'occurrence Auchan) doit tenir compte des conditions d'accès au site telles qu'elles peuvent être constatées de manière objective, sans que la circonstance qu'une partie de cette zone soit constituée de communes situées hors du territoire national ne justifie à elle seule leur exclusion, dès lors que la frontière qui les sépare ne constitue un obstacle ni géographique, ni monétaire à l'accès au site prévu; qu'il incombe en conséquence au demandeur de fournir, pour l'ensemble de la zone de chalandise ainsi définie, les renseignements prévus par le décret du 9 mars 1993 modifié et l'arrêté pris pour son application ». Extrait du $2^{\mathrm{e}}$ dossier de demande de création par transfert pour la CNEC, avril 2003.

13. Certains de ces effets frontières ont été développés dans Les zones d'activités: espaces pionniers à la périphérie des agglomérations, le cas lorrain, Colette Renard-Grandmontagne, Thèse soutenue à l’Université Nancy 2, décembre 2004, p. 349-373.

14. Le quartier du Val Saint-Martin construit dans les années 1960 dans le cadre d'une ZUP a été classé en ZRU (Zone de Redynamisation Urbaine) depuis 1996.

15. «Longwy se paie un cœur de 60 millions d'euros » et «Immochan et Frey signent un PAC pour l'avenir de l'agglomération » in Le Républicain Lorrain, 26 novembre 2004.

16. Le projet de la zone commerciale du Bivaque, à Longwy-Haut a été enterré par la réalisation de celui de Pôle Europe; voir l'article du Républicain Lorrain: "La zone commerciale du Bivaque définitivement aux oubliettes ", 19 décembre 2003.

17. La clientèle de l'hypermarché Auchan est constituée pour près de la moitié de clients belges et luxembourgeois, dans des proportions respectives de deux tiers et un tiers, soit exactement à l'inverse des prévisions.

18. La loi du 29 juin 1975 a été récemment abrogée par celle du 13 août 2004 relative à l'autorisation d'implantation commerciale. Cette dernière loi a été complétée par celle du 27 décembre 2005. Le caractère récent de ces deux textes législatifs ne remet pas en cause l'existence des différences réglementaires qui ont subsisté durant près de 30 ans entre les deux pays.

19. Rapport d'information d'Alain Fouché, sénateur de la Vienne, fait à la demande du Premier Ministre, Jean-Pierre Raffarin, sur l'évaluation du dispositif réglementaire garantissant l'équilibre entre les différentes formes de commerce, remis le 8 octobre 2004. Ce rapport comprend en annexe les principales dispositions réglementaires adoptées par les pays de l'Union Européenne. On pourra se reporter à la fiche $\mathrm{n}^{\circ} 3$ « Belgique » de l'annexe $\mathrm{C}$.

20. Expressions orales recueillies au cours d'interviews des acteurs locaux actuels dont certains ont traversé les 25-30 années de crise du bassin ; interviews réalisés en juillet 2004 et au printemps 2006. 
21. L'EPFL a succédé à l'EPML (Établissement Public de la Métropole Lorraine), créé par décret ministériel du 7 mars 1973. Il a longtemps été dirigé par J. Pierson décédé en 2005. Le nouveau Directeur Général nommé par le Ministre de l'Équipement, en septembre 2005, est Pascal Gauthier, issu du SGAR (Secrétariat Général aux Affaires Régionales). Depuis septembre 2001 sont membres du Conseil d'Administration, deux élus du bassin de Longwy, Messieurs Jacque et Ariès, ce dernier étant conseiller général du canton de Longwy. Jusqu'à cette date, il n'y avait pas de représentant de Longwy dans cette instance.

22. À titre de comparaison, le financement de la ZAC de Pompey-Frouard ( 90 ha), pour laquelle l'EPFL a été aussi maître d'ouvrage, s'est élevé à 22 millions d'euros. Le coût de l'aménagement de l'ensemble des zones d'activités du site de Pompey-Frouard est estimé à 40 millions d'euros.

23. Selon d'autres études effectuées en Lorraine, le prix du mètre carréde terrain commercial est 2 à 4 fois supérieur à celui du mètre carréde terrain industriel. Des valeurs de $30 € / \mathrm{m}^{2}$ contre $7,50 € / \mathrm{m}^{2}$ ont été citées pour Longwy, au début des années 2000 .

24. À titre de comparaison le financement du contrat territorial du Grand Nancy (400 000 personnes) s'élève à 93 millions d'euros.

25. De nombreux autres exemples peuvent être cités, comme Golbey dans l'agglomération d'Épinal ou Boulazac dans celle de Périgueux.

\section{RÉSUMÉS}

Le bassin de Longwy a déjà été l'objet de nombreuses publications depuis l'effondrement de la sidérurgie. Cet article qui n'est pas une synthèse d'un quart de siècle de reconversion, éclaire en revanche les réorientations récentes qui président maintenant au développement de ce territoire. Après une longue phase de reconversion industrielle prise en charge par les institutions nationales et européennes, aux résultats mitigés, vient de s'ouvrir une nouvelle ère. Celle-ci est résolument orientée vers un développement commercial et artisanal dont les acteurs sont à la fois des groupes de la grande distribution et les collectivités territoriales locales décidées à reprendre en main leur destin.

The Longwy basin has been the subject of numerous publications since the decline of the steel industry. This article is not a synthesis of a quarter of a century of reconversion, but sheds light on the recent reorientations which currently govern the development of this region. After a long period of industrial reconversion, underwritten, with mixed results, by national and European institutions, a new era is to begin. This one is orientated towards commercial development and craft industries, the players both retail groups and local and regional authorities determined to take their destiny into their own hands.

Seit dem Zusammenbruch der Stahlindustrie war das Revier Longwy bereits Gegenstand zahlreicher Veröffentlichungen. Dieser Artikel bildet keine Synthese des seit einem Vierteljahrhundert andauernden Umgestaltungsprozesses, sondern beleuchtet die jüngsten Neuorientierungen, welche die gegenwärtige Entwicklung dieses Raumes leiten. Nach einer langen Phase industrieller Umgestaltung unter der Leitung der nationalen und europäischen Institutionen mit mäßigen Ergebnissen, hat kürzlich eine neue Ära begonnen. Diese ist in 
starkem Maße ausgerichtet auf die Entwicklung des Handels und des Handwerks, deren Akteure gleichermaßen große Handelsunternehmen und die lokalen Gebietskörperschaften sind.

\section{INDEX}

Schlüsselwörter : Umstellung, Industriebrache, Aktivitätszone, Handelsentwicklung, Grosshandel, Grenze, Interkommunalität

Mots-clés : Reconversion, friche industrielle, zone d'activités, développement commercial, grande distribution, frontière, intercommunalité

Keywords : Reconversion, industrial relicts, trade area, commercial development, retail trade, border, intercommunality

\section{AUTEUR}

\section{COLETTE RENARD-GRANDMONTAGNE}

CERPA - Université de Nancy 2 - 23, bd Albert I ${ }^{\text {er }}-54015$ Nancy CEDEX 